\title{
A new covalent organic polymer used to highly selective detection of $\mathrm{Fe}^{3+}$ ions
}

\author{
Han Zhang ${ }^{1}$, Xin-Long Wang ${ }^{12, *}$, Chun-Yi Sun ${ }^{12}$, and Zhong-Min Su 12 \\ ${ }^{1}$ School of Materials Science and Engineering, Changchun University of Science and Technology, Changchun 130022, China. \\ ${ }^{2}$ National \& Local United Engineering Laboratory for Power Batteries, Key Laboratory of Polyoxometalate Science of Ministry of \\ Education Department of Chemistry, Northeast Normal University, Changchun 130024, China.
}

\begin{abstract}
Covalent organic polymers (COPs) received much attention due to their application in diverse fields, especially in sensing. A highly efficient fluorescence sensor in selective detection of $\mathrm{Fe}^{3+}$ ions is necessary to develop. In this work, we successfully synthesized a new covalent organic polymers named COP-TP, with the excellent performance in selective detection of $\mathrm{Fe}^{3+}$ ions, the quenching mechanism mainly due to the energy transfer process. The above results show COP-TP has the possibility to be a new candidate in selective detection as $\mathrm{Fe}^{3+}$ ions sensor.
\end{abstract}

\section{Introduction}

Covalent organic polymers (COPs) [1-2], as a new class of organic materials have drawn lots of attention due to their attractive properties with pre-disigned structure [3], stable-band [4], and good dispersion[5] as well as the chemical and physical stability. And the selected monomers or building units make the COPs have the possible applications in sensing, gas storage, chemical separation, drug delivery, and catalysis[6-10]. Fe3+ ions are the important trace elements in the body, which influence the oxygen-uptake and electron transfer[11-12]. Morever, the concentration of $\mathrm{Fe} 3+$ ions should be moderated at a suitable range, the deficiency and the overload would cause the diverse diseases. Different ions will cause different environmental and health problems, and the materials with specific and selective selection of $\mathrm{Fe} 3+$ ions are worthy to attention. To date, there are many materials used to act as sensors to detect metal ions, such as metal-organic polymers (MOPs) [13-16] and small organic molecules [17-20]. However, covalent organic polymers act as sensors to detect $\mathrm{Fe} 3+$ ions are rarely reported, a COP with excellent selective ability to $\mathrm{Fe} 3+$ ions is promising to design.

In this work, we successful designed and synthesized a new covalent organic polymer named COP-TP through the simple-green Suzuki-coupling reaction, characterized by FT-IR, PXRD, fluorescence spectrum and SEM. The COP has the great performance as sensor to selective detection of $\mathrm{Fe}^{3+}$ ions among the other trace elements ions and $\mathrm{Mg}^{2+}$ ions. The COP may provide a new fast method to selectively detect and monitor the $\mathrm{Fe}^{3+}$ in practice.

\section{EXPERIMENTAL SECTION}

\subsection{Materials and measurements}

All reagents and solvents for the synthesis were purchased from commercial sources and used as received, unless otherwise indicated. Infrared spectra were obtained from $\mathrm{KBr}$ pellets in a wavelength ranging from $3300-400 \mathrm{~cm}-1$ on a Nicolet 380 FT-IR spectrophotometer. PXRD pattern was recorded on a Siemens D5005 diffractometer with $\mathrm{Cu} \mathrm{K} \alpha(\lambda=1.5418$ $\AA$ ) radiation in the range of $2-15^{\circ}$. SEM images were recorded on XL-30 ESEM-FEG Scanning Electron Microscope.Photoluminescence spectra were recorded on a FL-4600 FL spectrophotometer.

\subsection{Synthesis of COP-TP}

The COP-TP was synthesized through the Suzukicoupling reaction according the literature (Scheme 1) [21], a mixture of Pyrene-2,7-diboronic acid, pinacol ester (PDABE) and 1,3,5-Tribromobenzene (TBB) (molar

\footnotetext{
* Corresponding author: wangx1824@nenu.edu.cn
} 


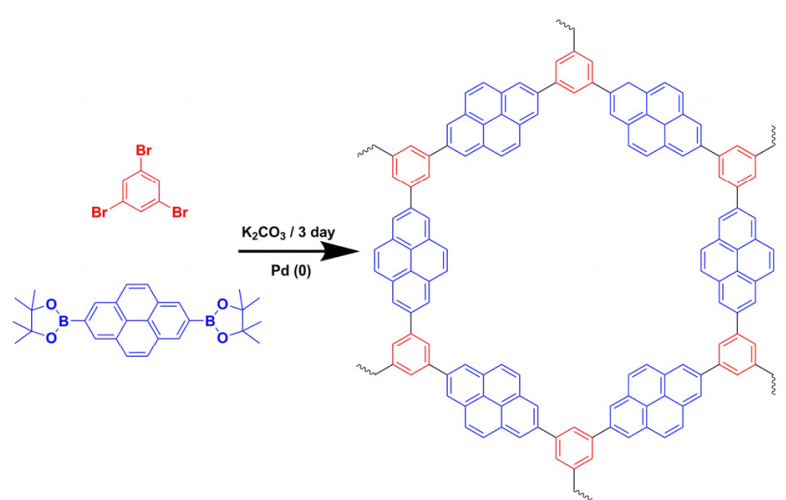

Scheme 1. Synthesis route of the COP-TP.

ratio: $3: 2)$, tetrakis(triphenylphosphine) palladium (0.01 $\mathrm{mmol}$ ) and $\mathrm{K}_{2} \mathrm{CO}_{3}(3.2 \mathrm{mmol})$ was dissolved in DMF / $\mathrm{H}_{2} \mathrm{O}(16 \mathrm{~mL} / 2 \mathrm{~mL})$, the mixture was degassed by three freeze-pump-thaw cycles and purged with $\mathrm{N}_{2}$. The mixture was stirred at $150{ }^{\circ} \mathrm{C}$ for $72 \mathrm{~h}$ and then cooled to room temperature and poured into water. The precipitate was collected by filtration, repeatedly washed by Soxhlet extraction for $24 \mathrm{~h}$, and finally dried in vacuum to give COP-TP as powder.

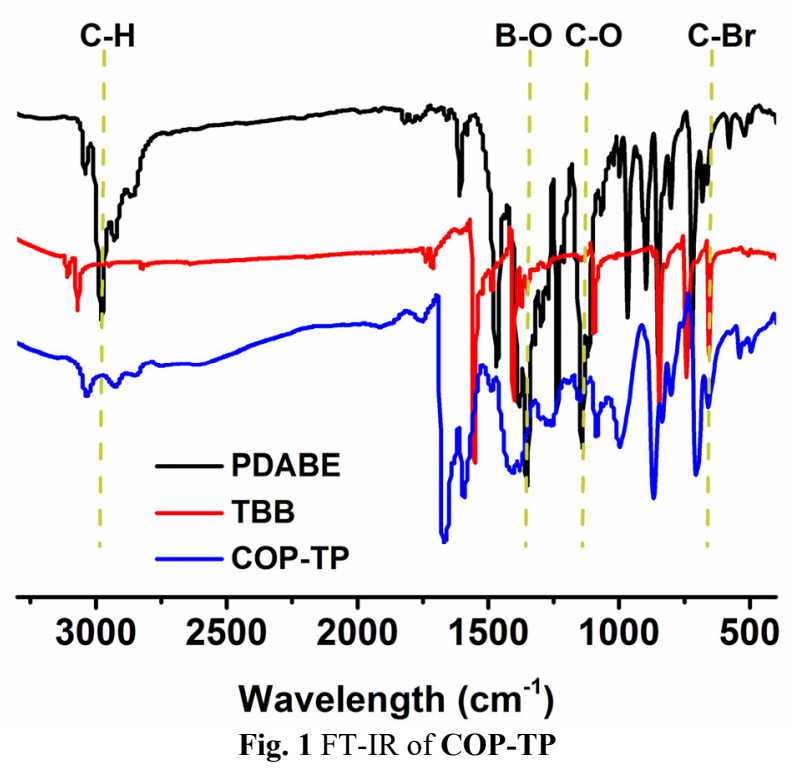

\subsection{Chemical sensing of metal ions}

Titration experiments of metal ions were carried out by adding same concentration of metal salt solutions $(20 \mu \mathrm{L})$ into the dimethylacetamide (DMA) suspension (1 mL) containing COP-TP $(0.1 \mathrm{mg} / \mathrm{mL})$ at intervals of $5 \mathrm{~min}$. Fluorescence spectra were recorded after the addition of metal salt solutions.

Fluorescence quenching $\%=(1-\mathrm{I} / \mathrm{I} 0) \times 100 \%$, where I0 is the initial fluorescence intensity in the absence of metal ions, I is the fluorescence intensity in the presence of corresponding analyte [22].

\section{Results and discussion}

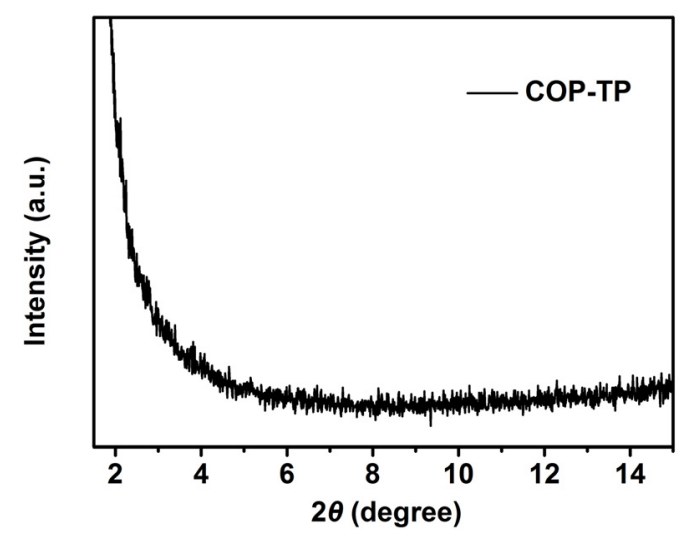

Fig. 2 PXRD of COP-TP

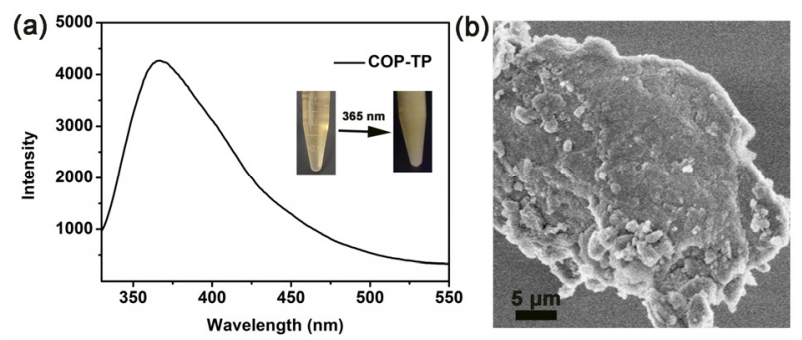

Fig. 3 (a) Fluorescence spectrum of COP-TP; (b) SEM image of COP-TP.

Fig. 1 shows the FT-IR spectra of the COP-TP , DBTPA and TBB monomers as the reference units. The direct evidence of the Suzuki coupling reaction has already occurred is that the disappearance of characteristic peaks at $615 \mathrm{~cm}-1$, belonging to the $\mathrm{C}-\mathrm{Br}$ vibration of the building monomers (TBB). And peaks at $1180 \mathrm{~cm}-1$ is also disappearance which belongs to the C$\mathrm{O}$ vibration of the building monomers (PDABE). The $\mathrm{B}-$ $\mathrm{O}$ vibration peaks around $1350 \mathrm{~cm}-1$ is a lot lower compare to the $\mathrm{PDABE}$, suggesting the $\mathrm{PDABE}$ was excessive before the reaction and the TBB was completed reacted. The spectra of FT-IR proved that the COP-TP was successful synthesized.

There is no obvious diffraction peak in the powder X-ray diffraction (PXRD) patterns (Fig. 2), indicating the amorphous nature of the COP-TP. The amorphous nature is similar to the reported materials that constructed by C-C coupling reaction [23]. The fluorescence spectrum (Fig. 3a) shows the COP-TP would emit the indigo color under the excitation wavelength at $300 \mathrm{~nm}$, and the photographs of COP-TP dispersed in DMA have a obvious change under the 365 nm UV-lamp. The SEM image (Fig. 3b) exhibits the COP-TP is a irregularly layered structure.

$\mathrm{Fe} 3+$ ions play the very important roles in oxygen uptake, 


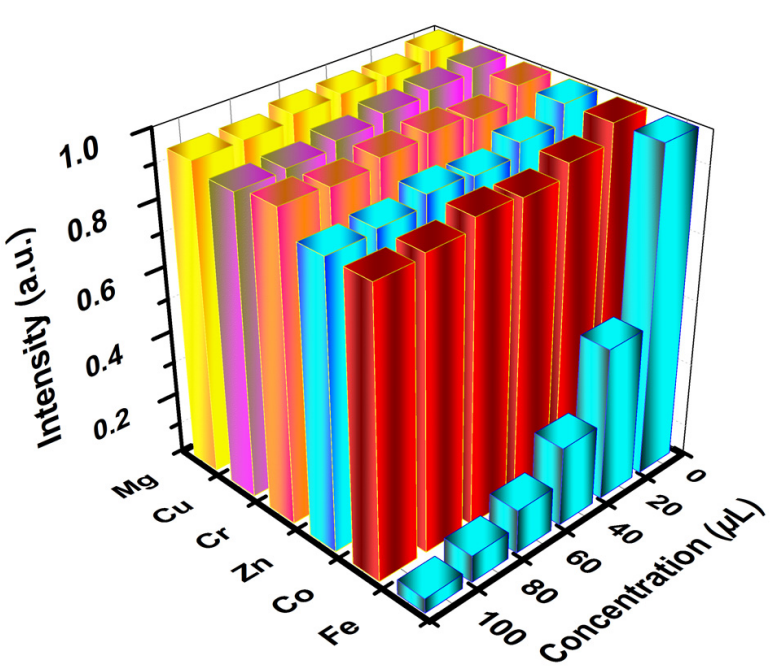

Fig. 4 Fluorescence intensity change of COP-TP after added different metal ions.

biological mechanisms and electronic transmission, and the moderate concentration is important to the life activity. The selective detection of Fe3+ ions among the other cation ions is very challenge and urgent. So we used the COP to act as a fluorescence sensor to detect $\mathrm{Fe} 3+$ ions. In order to get more insights into the metal ions sensing behavior of COP-TP, we tested the capability of them in the chemical sensing of metal ions include $\mathrm{Fe} 3+, \mathrm{Co} 2+, \mathrm{Zn} 2+, \mathrm{Cr} 3+, \mathrm{Cu} 2+, \mathrm{Mg} 2+$ ions. As shown in Fig. 4, the fluorescence intensity of COP-TP suspension has barely change after added $\mathrm{Co} 2+, \mathrm{Zn} 2+$, $\mathrm{Cr} 3+, \mathrm{Cu} 2+, \mathrm{Mg} 2+$ ions, and with the concentration up to $100 \mu \mathrm{L}$, the fluorescence intensity still not changed. The results showed the COP-TP will not be affected by the $\mathrm{Co} 2+, \mathrm{Zn} 2+, \mathrm{Cr} 3+, \mathrm{Cu} 2+, \mathrm{Mg} 2+$ ions. As for $\mathrm{Fe} 3+$ ions, when added $20 \mu \mathrm{L} \mathrm{Fe} 3+$ ions to the suspension of COP-TP, the quenching effect is very obvious. And with the increase of the $\mathrm{Fe} 3+$ ions concentration, the fluorescence intensity of COP-TP decreased gradually, the phenomenon exhibits the COP-TP has the excellent performance to selective detection of $\mathrm{Fe} 3+$ ions.

The quenching percentage can directly express the selective expression of Fe3+ ions. As shown in Fig. 5, the quenching percentage of COP-TP is up to the 95.41\% after added $100 \mu \mathrm{L} \mathrm{Fe} 3+$ ions, whereas the fluorescence quenching percentage after added $\mathrm{Co} 2+$, $\mathrm{Zn} 2+, \mathrm{Cr} 3+, \mathrm{Cu} 2+, \mathrm{Mg} 2+$ ions are $12.76 \%, 12.76 \%$, $6.00 \%, 8.05 \%, 5.44 \%$, respectively. The results show the COP-TP has the great selective ability to detect Fe3+ ions in aqueous.

The quenching process of COP-TP after add Fe3+ ions mainly due to the energy transfer mechanism. As previous reported [24-26], the Fe3+ ions has the empty d orbital, which is the perfect receptor to electrons. And the fluorescence COP is a electrons donor material, when the UV-lamp excites the electrons from COP-TP, the electrons would transfer to the $\mathrm{d}$ orbital with the energy transfer, leading to the fluorescence quenching. This clear mechanism could provide a theoretical basis for the successful application of material.

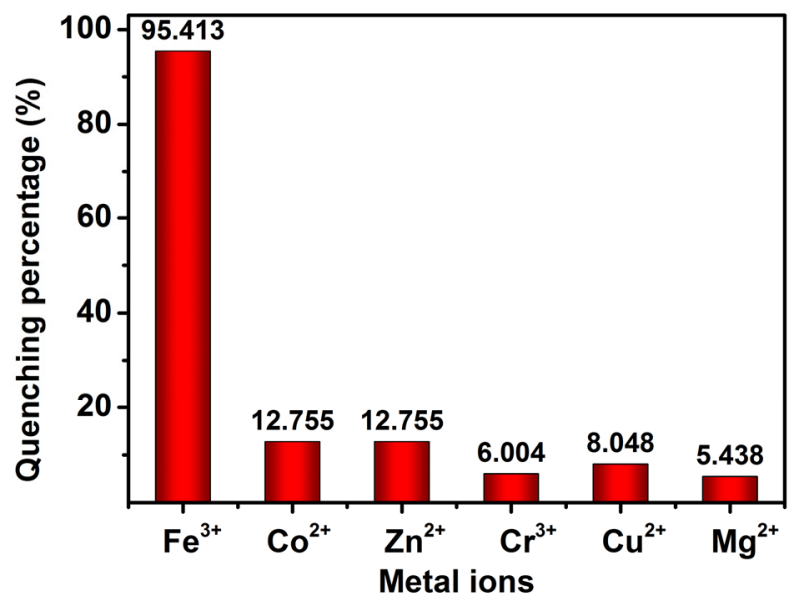

Fig. 5 Quenching percentage of COP-TP after added $100 \mu \mathrm{L}$ metal ions.

\section{Conclusion}

We successful synthesized a new covalent organic polymers named COP-TP by Suzuki reaction, and confirmed by FR-IR, PXRD, Fluorescence spectrum, and SEM. The emission color change under the UVLamp shows the potential application in sensing, and the selective tests show the COP-TP has the great performance in selective detection of $\mathrm{Fe}^{3+}$ ions. And The clear mechanism provides a theoretical basis for its application. The COP-TP may provide a new method to selective detection of $\mathrm{Fe}^{3+}$ ions in aqueous.

\section{References}

1. Valmik P. Jejurkar, Gauravi Yashwantrao and Satyajit Saha, New J. Chem., 44 (2020).

2. Weijie Zhang, Briana Aguila and Shengqian Ma J. Mater. Chem. A, 5 (2017).

3. Ruth Gomes, Piyali Bhanja and Asim Bhaumik, Chem. Commun., 51 (2015).

4. Jeehye Byun, Sang-Hyun Je, Hasmukh A. Patel, Ali Coskun and Cafer T. Yavuz,J. Mater. Chem. A, 2 (2014).

5. Zhonghua Xiang, Dapeng Cao and Liming Dai,Polym. Chem., 6 (2015).

6. Zhonghua Xiang and Dapeng Cao, J. Mater. Chem. A, 1 (2013).

7. Han Wang, Hui Wang, Ziwei Wang and Junwang Tang, Chem. Soc. Rev., 49 (2020).

8. Huiqi Wang, Zheng Li, Wei Feng and Qiong Jia, New J. Chem., 41 (2017).

9. Lin Guo, Meng Wang, Xiaofei Zeng and Dapeng Cao, Mater. Chem. Front., 1 (2017).

10. Yao Nie, Li Li and Zidong Wei, Chem. Soc. Rev., 44 (2015).

11. T. Ohmori, H. Takahashi, H. Mametsuka and E. Suzuki, Phys. Chem. Chem. Phys., 2 (2000). 
12. Wenjuan Han, Ming Lu, Junnan Chen, Haojie Li, Haibo Li, Bingsen Zhang, Wei Zhang and Weitao Zheng, J. Mater. Chem. A, 8 (2020).

13. Midhun Mohan, Sanil Rajak, Alexandre A. Tremblay, Thierry Maris and Adam Duong, Dalton Trans., 48 (2019).

14. Xiong Li, Jianwei Guo, Hangbo Yue, Jiawei Wang and Paul D. Topham, RSC Adv., 7 (2017).

15. Nazir Ahmad, Hussein A. Younus, Adeel H. Chughtai and Francis Verpoort, Chem. Soc. Rev., 44 (2015).

16. Zexing Wu, Jie Wang, Kedong Xia, Wen Lei, Xien Liu and Deli Wang, J. Mater. Chem. A, 6 (2018).

17. Xu-dong Wang and Otto S. Wolfbeis, Chem. Soc. Rev., 43 (2014).

18. Xiangcheng Sun, Ying Wang and Yu Lei, Chem. Soc. Rev., 44 (2015).

19. Sankarasekaran Shanmugaraju and Partha Sarathi Mukherjee, Chem. Commun., 51 (2015).

20. Guiqian Fang, Hao Wang, Zhancun Bian, Jie Sun, Aiqin Liu, Hao Fang, Bo Liu, Qingqiang Yao and Zhongyu Wu, RSC Adv., 8 (2018).

21. Han Zhang, Jie Zhou, Guo-Gang Shan, Guang-Fu Li, Chun-Yi Sun, Dong-Xu Cui, Xin-Long Wang and Zhong-Min Su, Chem. Commun., 55 (2019).

22. Guangfu Li, Dongxia Zhu, Xinlong Wang, Zhongmin $\mathrm{Su}$ and Martin R. Bryce, Chem. Soc. Rev., 49 (2020).

23. Jinqiao Dong, Anil Kumar Tummanapelli, $\mathrm{Xu} \mathrm{Li}$, Shaoming Ying, Hajime Hirao, and Dan Zhao, Chem. Mater. 28 (2016).

24. Naresh Kumar, Vandana Bhalla and Manoj Kumar, Analyst, 139 (2014).

25. Terianna J. Wax and Jing Zhao, J. Mater. Chem. C, 7 (2019).

26. William P. Lustig, Soumya Mukherjee, Nathan D. Rudd, Aamod V. Desai, Jing Li and Sujit K. Ghosh, Chem. Soc. Rev., 46 (2017). 